\title{
Spontaneous Resolution of a Relapsed Right Neck Mass due to Sternocleidomastoid Hypertrophy in a Congenital Muscular Torticollis Infant
}

\author{
Myongsoon Sung ${ }^{1}$, Jonghyun Lee', Sung Soo Kim', Kyung Hwan Byun² \\ 'Department of Pediatrics, Soonchunhyang University Gumi Hospital; ${ }^{2}$ Department of Radiology, CHA Gumi Medical Center, CHA University School of \\ Medicine, Gumi, Korea
}

\begin{abstract}
There was an episode of complete recovery of congenital muscular torticollis presenting an ipsilateral neck mass due to the right sternocleidomastoid muscle (SCM) with other symptoms such as contralateral rotation of the head and restricted motion following initial physiotherapy for 6 months in a male infant. Physical examination and neck ultrasonography in the neonate clinic at 6 months revealed that his neck mass had disappeared, so therapy was discontinued. After 12 months of the episode, there was a reoccurrence of congenital muscular torticollis with the only symptom of ipsilateral neck mass and subsequently recovered without restricted motion. After 26 months of the reoccurrence, with no treatment except for 2 months of the second physiotherapy which was proven to be ineffective, spontaneous resolution of the right neck mass was demonstrated on serial ultrasound. Recovery of relapse SCM hypertrophy is extremely rare; therefore, we report the case. We suggest that the case may help clinicians diagnose and treating patients with the recovery of relapse SCM hypertrophy without complications.
\end{abstract}

Keywords: Torticollis; Congenital sternocleidomastoid muscle; Newborn infant

\section{INTRODUCTION}

Congenital muscular torticollis (CMT), one of the most common musculoskeletal disorders in infancy, is a neck deformity that involves contracture of the sternocleidomastoid muscle (SCM) [1]. The incidence of CMT varies from $0.008 \%$ to $3.92 \%$ in all newborns, and its characteristic clinical features include ipsilateral tilting of the head, contralateral rotation of the face and chin, and a palpable neck mass [1]. Concerning prognosis, early diagnosis is important to begin rehabilitation therapy as early as possible [2]. With physical therapy, $>90 \%$ of infants with CMT fully recover with a good prognosis [3]. Nonetheless, in some children with atypical clinical features, CMT is diagnosed at a later time, and late diagnosis, the persistence of contracture of the SCM despite physical therapy, plagiocephaly, or scoliosis is a poor prognostic factor.

Although some refractory cases have been reported, there has been no report of CMT cases with complete recovery followed by a relapse of SCM hypertrophy and subsequent recovery. Thus, our case report is to describe the complete recovery of CMT followed by relapsed SCM hypertrophy and recovery without complications in a pediatric male patient.

\section{CASE REPORT}

A newborn boy with no family history of torticollis was delivered via a cesarean section, because of a breech position at $37+3$ weeks of gestation to a primigravida woman with gestational diabetes mellitus in March 2014. His birth weight was 2,660 g, birth height was $49 \mathrm{~cm}$, and head circumference was $34.5 \mathrm{~cm}$. His Apgar score was 7/9 because of the difficult delivery of breech position in a baby; after 2 hours of close observation, his condition improved and he was discharged in the neonate unit according to the usual schedule. However, he was found to have a right neck mass, contralateral rotation of the face, and right-sided restricted motion at the outpatient neonate clinic at age 16 days. We observed asymmetrical thickening, contour bulging, and increased internal echogenicity of the right SCM on neck ultrasonography at age 18 days
Correspondence to: Myongsoon Sung

Department of Pediatrics, Soonchunhyang University Gumi Hospital, 179 1(il)gongdan-ro, Gumi 39371, Korea

Tel: +82-54-468-9114, Fax: +82-54- 468-9114, E-mail: 89738@schmc.ac.kr

Received: Jul. 24, 2020 / Accepted after revision: Oct. 7, 2020
(C) 2020 Soonchunhyang Medical Research Institute This is an Open Access article distributed under the terms of the Creative Commons Attribution Non-Commercial License (https://creativecommons.org/licenses/by-nc/4.0/) 
(diameter: $1.07 \mathrm{~cm}$ right side of SCM, $0.46 \mathrm{~cm}$ left side of SCM) (Fig. 1A, B). Thus, the right CMT was diagnosed, pediatrics referred to the rehabilitation department, and the patient underwent physical therapy 3 times a week at the rehabilitation center.

After 6 months of stretching exercises, he was able to move his neck freely without right-sided restricted motion or contralateral rotation of the face. Moreover, no neck mass was seen in the right neck on the physical examination of the neonate specialist. We repeated neck ultrasonography in September 2014 (at age 6 months), and found the symmetrical thickness of the left and right SCM with no contour bulging in the right SCM (diameter: $0.44 \mathrm{~cm}$ right side of SCM, $0.49 \mathrm{~cm}$ left side of SCM) (Fig. 1C, D). Therefore, physical therapy was discontinued, and the patient was scheduled for follow-up visits.

He returned to our outpatient pediatric clinic with a right neck mass (round and non-fixed and skin redness) without fever, in September 2015 (at age 18 months) (Fig. 2). Physical examination revealed that the neck mass was soft and regular margin and there was no restricted neck motion, facial asymmetry, or plagiocephaly to the boy. We performed neck ultrasonography, which showed asymmetrical thickening of the left and right SCM and relapse of contour bulging in the right SCM (diameter: $0.70 \mathrm{~cm}$ right side of SCM, $0.52 \mathrm{~cm}$ left side of SCM) (Fig. 3A, B).

Pediatrics referred to the rehabilitation department, and stretching exercises were performed again twice a week for 2 months at the rehabilitation center and every day at home by the boy's parents. However, the neck mass did not show any change thus for 2 months of the second physiotherapy which was proven to be ineffective were stopped, but there was no restriction of neck motion and posture of the body. Regular physical examinations were conducted in the clinic and we repeated neck ultrasonography on August 2016 (diameter: $0.66 \mathrm{~cm}$ right side of SCM, $0.46 \mathrm{~cm}$ left side of SCM) and April 2017 (diameter: $0.58 \mathrm{~cm}$ right side of SCM, $0.37 \mathrm{~cm}$ left side of SCM), but no changes were noted compared with the previ-
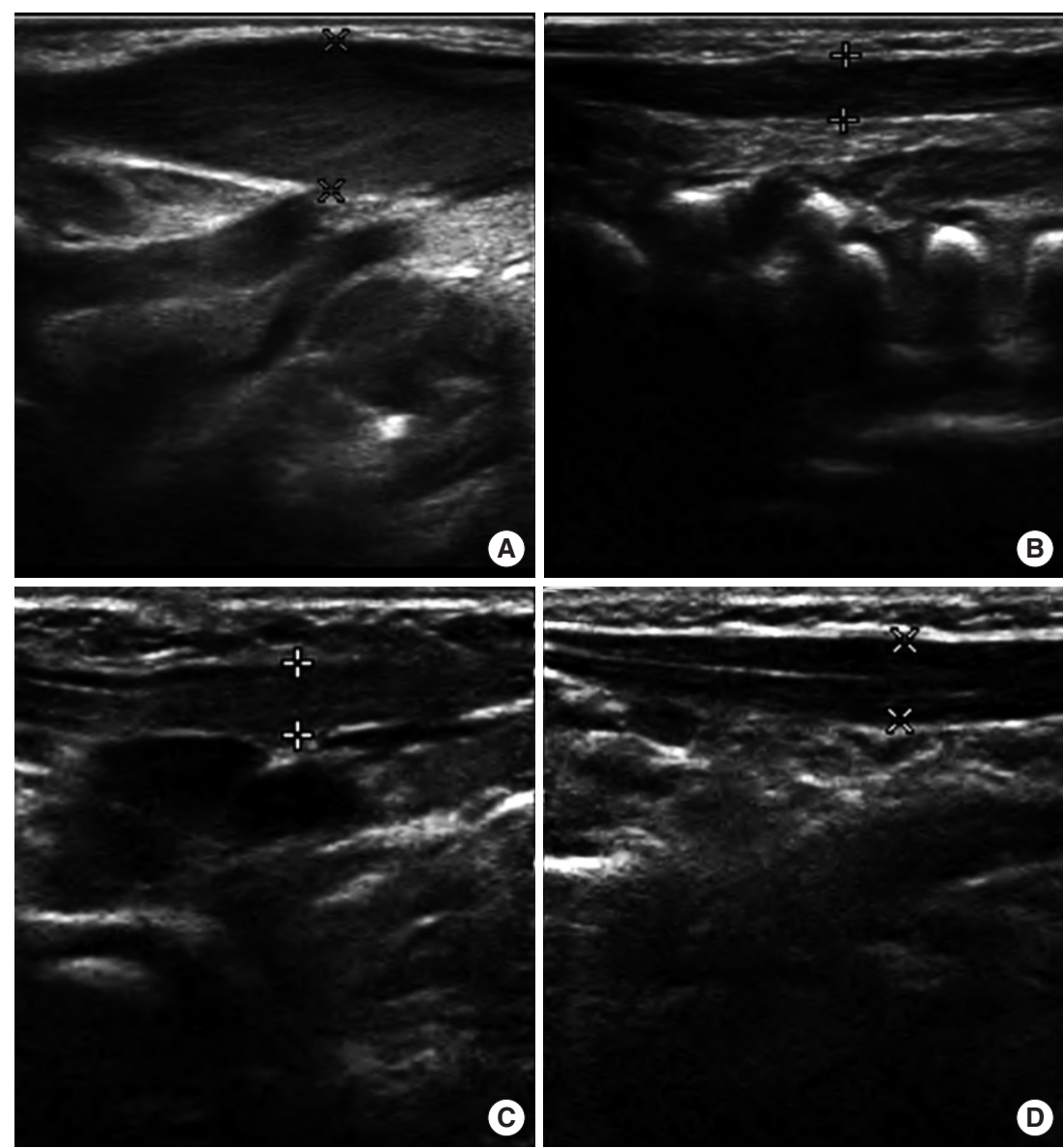

Fig. 1. Neck ultrasonography images in March 2014 (A, B) and September 2014 (C, D). (A) Right side of SCM (1.07 cm). (B) Left side of SCM (0.46 cm). (C) Right side of SCM $(0.44 \mathrm{~cm})$. (D) Left side of SCM $(0.49 \mathrm{~cm})$. SCM, sternocleidomastoid muscle. 


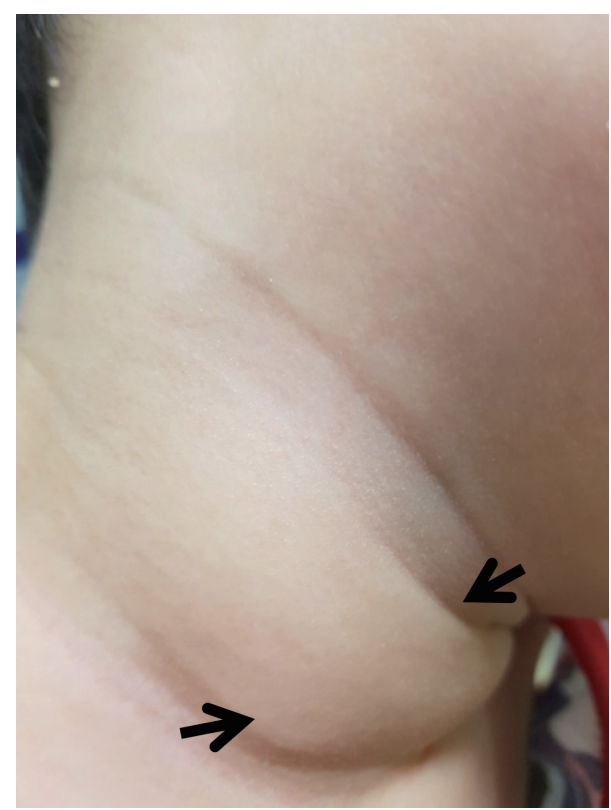

Fig. 2. Revisit in September 2015. Right neck mass (arrows). ous neck scans.

Eventually, the right neck mass disappeared and no restriction of motion was noted. Ultrasonography showed the return of a symmetrical thickness of the left and right SCM and no contour bulging in the right SCM (diameter: $0.61 \mathrm{~cm}$ right side of SCM, $0.60 \mathrm{~cm}$ left side of SCM) on January 2018 (at age 34 months) (Fig. 3C, D) and this status had been continued on September 2019 (at age 54 months). All neck scans were conducted by the same radiologist.

The patient's caregiver provided written informed consent for the publication of clinical details and images.

\section{DISCUSSION}

CMT is the third most common congenital musculoskeletal disorder after hip dysplasia and clubfoot [1]. Although its etiology remains unclear, there are some well-substantiated hypotheses,
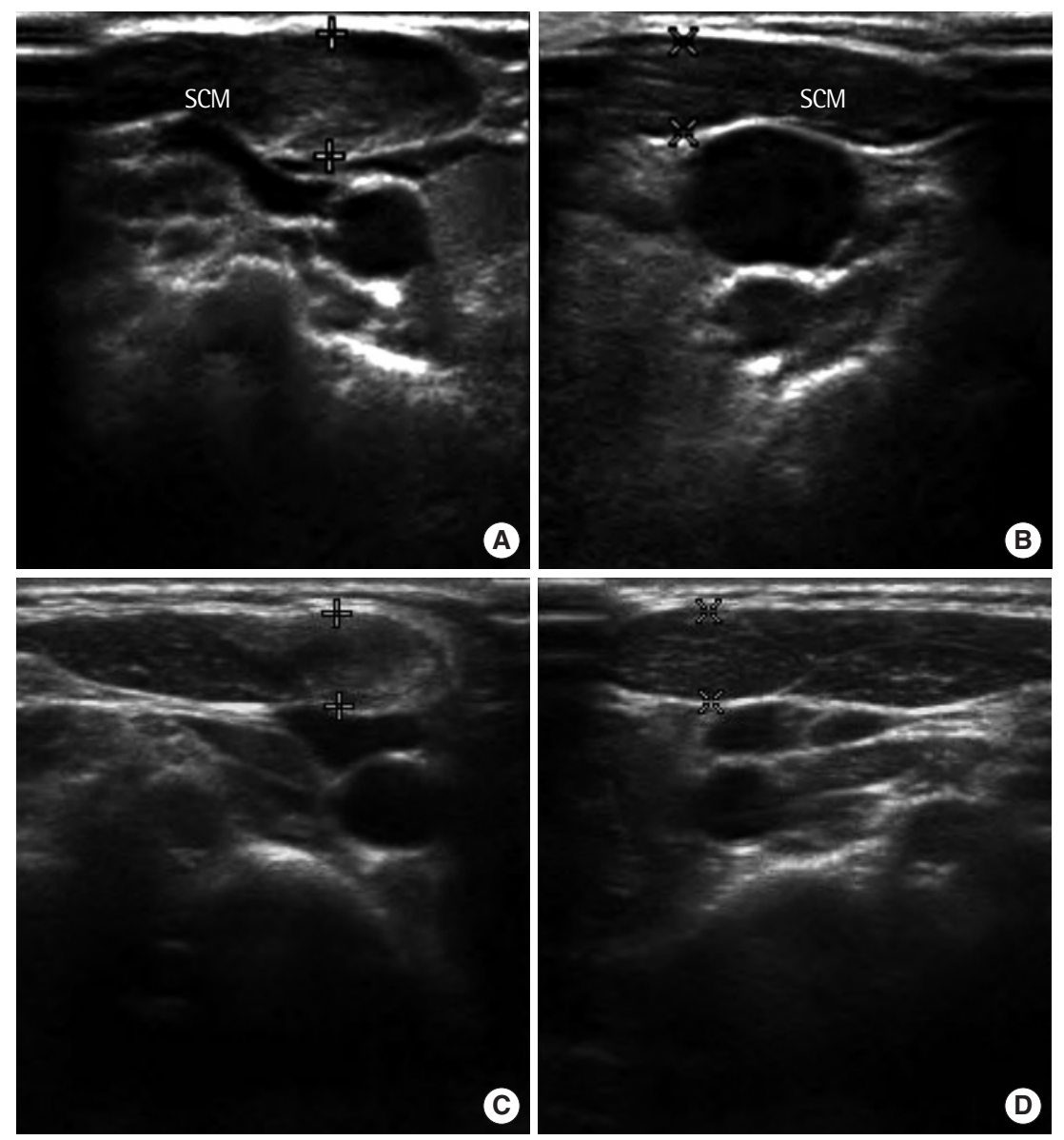

Fig. 3. Neck ultrasonography images taken in September 2015 (A, B) and January 2018 (C, D). (A) Right side of SCM (0.70 cm). (B) Left side of SCM (0.52 cm). (C) Right side of SCM (0.61 cm). (D) Left side of SCM $(0.60 \mathrm{~cm})$. SCM, sternocleidomastoid muscle. 
including intrauterine malposition, which causes an ischemic event and birth-trauma [2]. CMT may be classified into three types (postural torticollis, muscular torticollis, and SCM tumor or pseudotumor) and may present at birth or 2-4 weeks after birth $[1,2]$.

Early diagnosis in the neonate period is important for predicting prognosis and beginning rehabilitation therapy [2], so a careful physical examination of the neonate period was necessary. Typical CMT is diagnosed and has a good prognosis; however, these are not true for atypical CMT. According to a report, $>98 \%$ of infants with torticollis treated before 1 month of age recover by 2.5 months of age. Infants from 1 to 6 months usually require about 6 months of treatment and it is possible that torticollis will resolve spontaneously, but infants after 6 months could require from 9 to 10 months of treatment, with progressively fewer infants achieving normal range [4]. However, the chance of relapse is possible in any case. Unfortunately, the incidence of spontaneous resolution of CMT has been unknown, and there are no documented protocols to predict who will solve it in a more serious or sustained form [4]. Therefore, infants should be reassessed by their physical therapist or other providers 3-12 months after their symptoms have resolved as well as our boy case [4].

However, SCM contracture can persist despite physical therapy, with consequent craniofacial asymmetry, plagiocephaly, or scoliosis. Surgical intervention may be indicated in refractory cases, but only if the child does not respond to conservative treatment for 12 months [5,6]. Some studies have reported on the use of botulinum toxin injections in patients who still had a considerably shortened SCM, despite performing stretching exercises [7]. Injected botulinum toxin causes SCM paralysis, facilitating the stretching of the SCM, and decreasing the muscle mass. However, our patient showed complete recovery followed by a relapse of SCM hypertrophy with no motion restriction. Thus, we did not administer botulinum toxin injection in this case. In another case report, three infants experienced CMT relapse after the follow-up visit 1month after full resolution but achieved complete resolution after three cycles of physical therapy [2]. Therefore, infants need an observation period, during which the rehabilitation plan is continuously implemented and the head posture is checked until the resolution is stable.

The prognostic factors are early stretching exercises for children with CMT diagnosed before the age of 3 months and a difference in SCM thickness between the affected and normal sides [8,9]. In our case, the diagnosis and initiation of stretching exercises occurred before 1 month of age, neonate period, and the difference in SCM thickness was not large on initial ultrasonography. We demonstrated that relapse of SCM hypertrophy may occur in patients with CMT without poor prognostic factors.

CMT should be checked at regular intervals even if recovery is complete after rehabilitation treatment. Our report describes a rare case of complete recovery, relapse, and recovery of SCM hypertrophy without complications in a boy from neonate to 34 months old. Our findings may help clinicians diagnose and treat relapsed SCM hypertrophy without complications.

\section{REFERENCES}

1. Chen MM, Chang HC, Hsieh CF, Yen MF, Chen TH. Predictive model for congenital muscular torticollis: analysis of 1021 infants with sonography. Arch Phys Med Rehabil 2005;86:2199-203.

2. Carenzio G, Carlisi E, Morani I, Tinelli C, Barak M, Bejor M, et al. Early rehabilitation treatment in newborns with congenital muscular torticollis. Eur J Phys Rehabil Med 2015;51:539-45.

3. Collins A, Jankovic J. Botulinum toxin injection for congenital muscular torticollis presenting in children and adults. Neurology 2006;67:1083-5.

4. Kaplan SL, Coulter C, Sargent B. Physical therapy management of congenital muscular torticollis: a 2018 evidence-based clinical practice guideline from the APTA Academy of Pediatric Physical Therapy. Pediatr Phys Ther 2018;30:240-90.

5. Sonmez K, Turkyilmaz Z, Demirogullari B, Ozen IO, Karabulut R, Bagbanci B, et al. Congenital muscular torticollis in children. ORL J Otorhinolaryngol Relat Spec 2005;67:344-7.

6. Wei JL, Schwartz KM, Weaver AL, Orvidas LJ. Pseudotumor of infancy and congenital muscular torticollis: 170 cases. Laryngoscope 2001;111(4 Pt 1):688-95.

7. Ta JH, Krishnan M. Management of congenital muscular torticollis in a child: a case report and review. Int J Pediatr Otorhinolaryngol 2012;76: 1543-6.

8. Lim D, Kwon W, Cha SW, Yoo H, Lim S, Park JM, et al. The sonographic correlation between the sternocleidomastoid muscle thickness and the prognosis of congenital muscular torticollis. J Korean Soc Radiol 2009;60: 133-8.

9. Han JD, Kim SH, Lee SJ, Park MC, Yim SY. The thickness of the sternocleidomastoid muscle as a prognostic factor for congenital muscular torticollis. Ann Rehabil Med 2011;35:361-8. 